\title{
Effect of reference number on magnitude estimation
}

The psychophysical function obtained by the method of magnitude estimation was influenced by the reference number(modulus) assigned to a "standard" line and the position of this standard in the range of comparison stimuli. Data from two experiments with judgments of apparent length of lines show how both variables systematically affect the slope of the power function. Allowing $O$ to choose his own reference numbers, even though these numbers varied among Os, tended to produce less variability in slope than if $\mathrm{E}$ imposed fixed reference numbers for 0 to use.

In psychophysical judgments, systematic effects may be a direct result of perceptual or context variables related to the stimulus, or they may indirectly result from the enforced use of particular response choices. For ease of data handling most experiments severely limit an O's set of responses while also claiming that this response restriction is helpful in minimizing systematic response effects. However, the use of arbitrary and often circumscribed response choices can introduce a bias that passes unremarked unless alternative response choices are required for the same judgments. In practice, though, experiments that vary response choices are seldom performed.

In the first experiment of this investigation lengths of viewed lines were estimated with a different number attached to a single reference line in each condition. Thus the magnitude estimates open to a subject were limited by the magnitude of the arbitrary reference number. It was hypothesized that the use of different reference numbers selected by $\mathbf{E}$ would produce systematic judgment differences owing to the fact that Os do not manipulate all numbers in our decimallybased system with equal facility. By contrast, in a second experiment it was hypothesized that a "free modulus" condition in which $O$ himself selected the response modulus would tend to reduce judgment differences across the range of different number assignments since $O$ would select numbers that he could manipulate with facility. If the hypotheses in both experiments were borne out, free variation in 'number use in the second experiment would minimize judgment error as compared to the "more standardized" response conditions of the first experiment.

\section{Method}

The stimuli were lines $1.75,2.80,3.85,5.775,7.70$, $11.025,14.00,17.675,22.225$, and $26.25 \mathrm{in}$. long. The independent variables were: (1) reference number (or modulus) assigned to the standard stimulus, i.e., 1, 7, 14,100 , and 1000, including a no-modulus or free modulus condition; and (2) the magnitude of the standard
$.875,1.75,5.775,14.00,26.25$, and 43.00 in. This range permitted a possible interaction between the magnitude of the reference number and the magnitude of the standard stimulus to be evaluated. The variable of main interest was the reference number assigned to the standard stimulus by the $\mathbf{E}$, or the number assigned to the first stimulus by the $O$ when the free-modulus variation of the method of magnitude estimation was used; the chief dependent variable was the slope of the psychophysical function. Judgments of apparent length of lines were obtained because these had been shown in pilot experiments to conform to the simple form of the power function.

The lines were projected on a screen for group presentation. The order of presentation of lines was irregular with each line presented twice. Every $O$ in every group received the same order. Judgments were obtained from different groups of 15 to 20 college freshmen and sophomores without any previous experience in psychophysical experiments. All groups of Os were run in the same experimental room under the same conditions of illumination, etc. The same instructions and examples were used for all groups.

\section{Results and Discussion}

Geometric means of the responses to each of the 10 stimuli were computed for all groups. The simple power function, $\psi=k S^{n}$, fits the data. Figure 1 shows

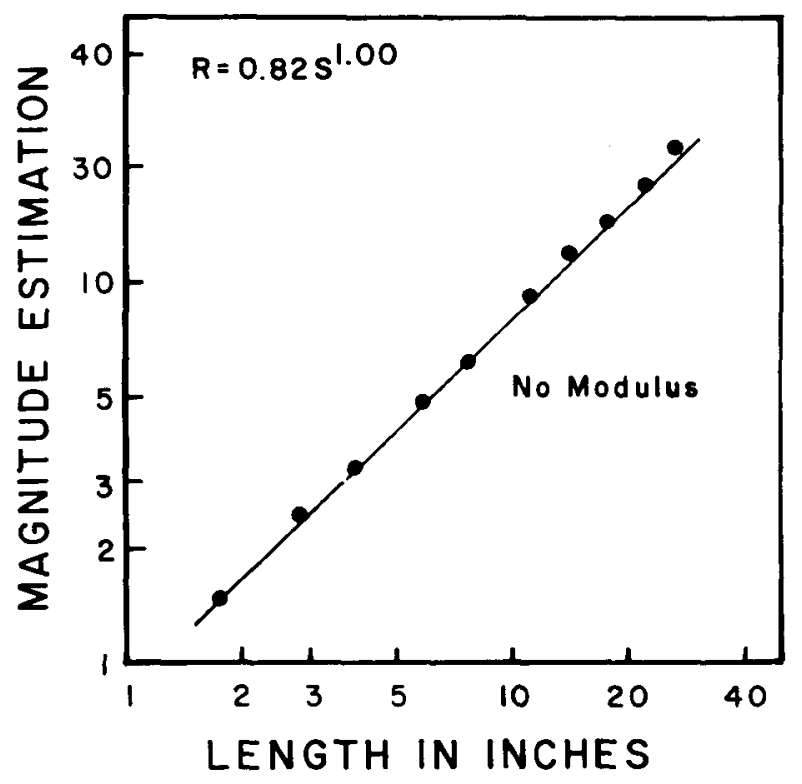

Fig. 1. Magnitude estimation as a function of line-length plotted in logarithmic coordinates. (Line fitted by method of least squares.) 


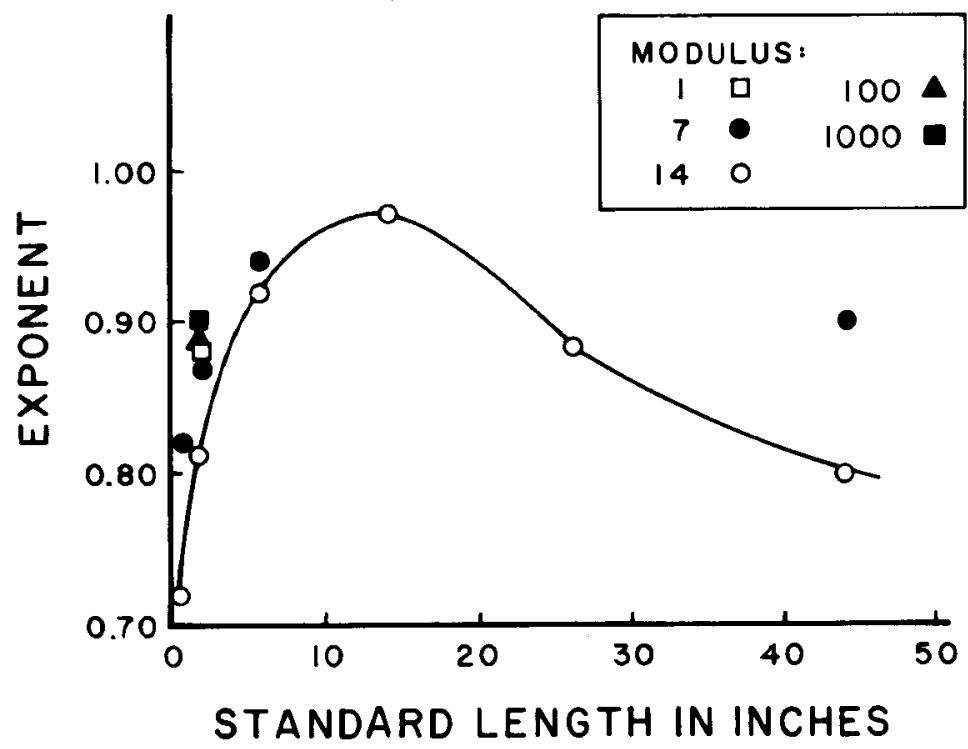

Fig. 2. The slope of the power function in relation to the position of the standard in the comparison series and the size of the reference number assigned by $E$. (Curve fitted by eye.)

the results plotted in $\log -\log$ coordinates for the group in which no modulus was specified.

The results for the groups for which a modulus was specified are summarized in Fig. 2 where the exponent of the power function is plotted as a function of the magnitude of the reference number and the position of the standard in the comparison series. First of all, these results support an earlier experiment (Engen \& Levy, 1955) in showing that the position of the standard stimulus, regardless of numerical value, has a consistent effect. The steepest slope (largest exponent) is obtained for standards in the middle of the stimulus series, and the slope decreases as the value of standard is either increased or decreased.

In addition, the results show that the lower the reference number the larger the exponent. However, it should be noted that this generalization must be qualified by the result that 1,100 , and 1,000 produce nearly identical results. Apparently the $O$ ignores zeros in this situation, but he tends otherwise to use a wider range of numbers when assigned a lower reference number (i.e., 1 versus 7 and 7 versus 14). There is some evidence of an interaction between the magnitude of the reference number and the magnitude of the standard stimulus since the slope varies less with reference number 7 (although no data were obtained for standard values 14.00 and 26.25) than with reference number 14. This difference is especially noticeable where standard lines were shorter or longer than the range of comparison stimuli.

The results support the working assumption of this research that the numerical values the $O$ is required to work with will tend to alter the psychophysical function. The alteration seems to be small but reliable, and it therefore does not necessarily invalidate the assigned use of numbers in psychophysical scaling. For example, even the lowest exponent does not approach that for loudness, nor does the highest ex- ponent approach that for heaviness (Stevens, 1957). There may indeed be something invariantabout psychophysical function after the variance due to numerical biases has been removed. Is it possible to remove or decrease this bias?

One possibility is the free-modulus variation of the method in which the $O$ himself determines the range of numbers he works with. We obtained such data for another $80 \mathrm{Os}$ in an experiment like the one shown in Fig. 1 obtained with a free modulus. In this condition the $O$ himself had to decide what number was subjectively appropriate to the first line presented, which was 5.75 in. in all conditions, then assign additional numbers to the remaining lines. After the experiment, the Os were arbitrarily divided into five categories on the basis of the numbers they assigned to the shortest line the first time it was presented. The numbers in each category were: $1 / 4$ to $3 / 4,1,2,3$ and 4 , and 5 to 16 inclusive; Ns were $5,28,23,17$, and 7 respectively. A power function was fitted to the data for each group, and the exponent of the function is plotted as a function of the size of the reference number in Fig. 3. Except for the Os in the first category with the smallest $\mathrm{N}$ (who imposed the difficulty of manipulating fractional values on themselves), Os produced very nearly the same psychophysical function despite variation in the reference number. It appears, therefore, that while there is a context effect associated with the magnitude of the reference number assigned by the $E$, this effect can generally be eliminated or sharply reduced by allowing the $O$ to choose his own modulus from the start.

Another approach is to predetermine the appropriate average subjective numerical value of the standard. The exponent for apparent length (Stevens, 1957) is near 1.00, and Fig. 2 shows that except for the freemodulus condition the exponent closest to this value was obtained when the number 14 was assigned by the 


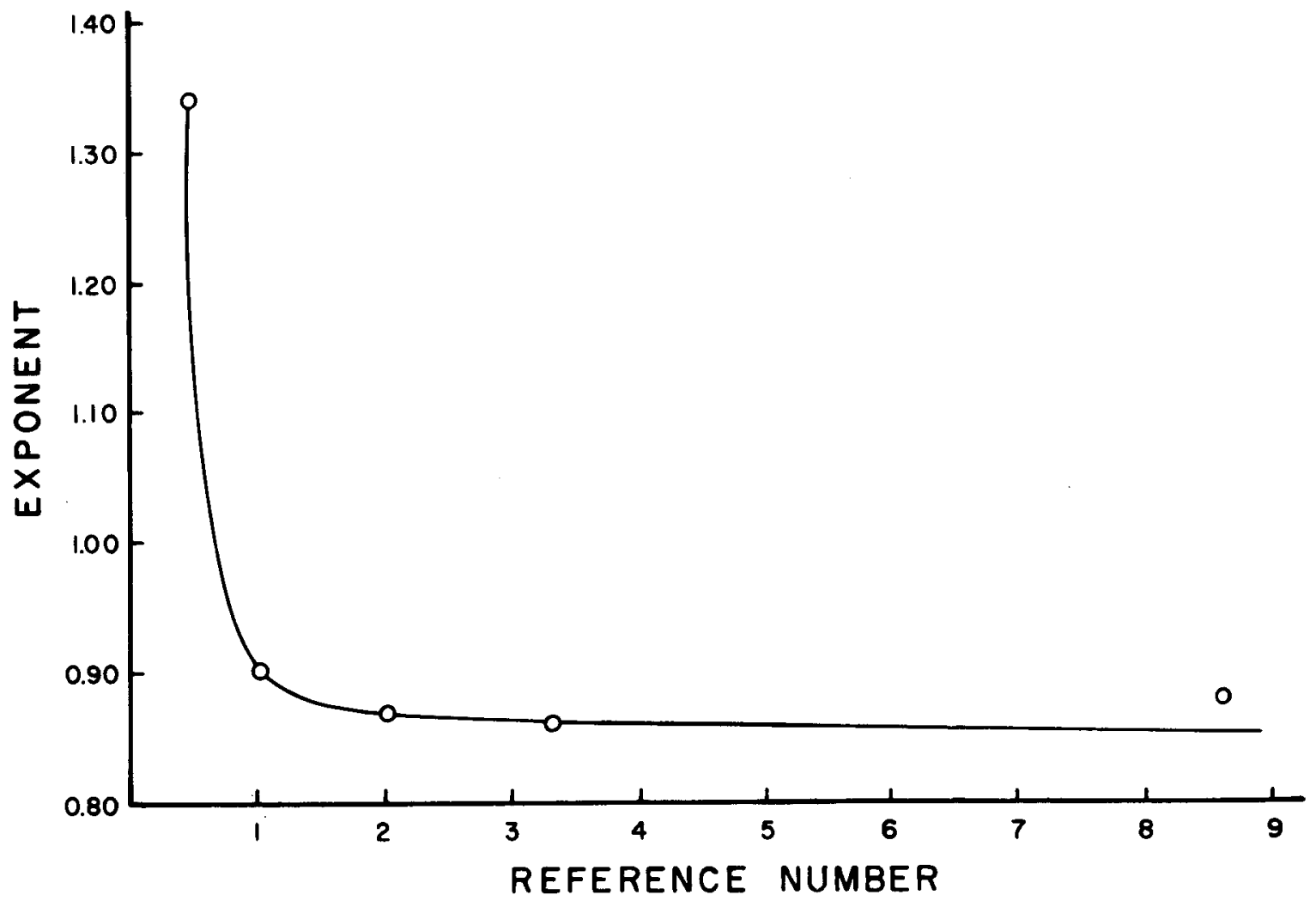

Fig. 3. The slope of the power function in relation to the size of the reference number selected by 0 . Values plotied on abscissa are means used for the smallest stimulus in each category. (Curve fitted by eye.) See text for details.

$\mathrm{E}$ to a line that was actually $14 \mathrm{in.}$ long and 7 to a line that was close to 7 in. long.

It seems reasonable to conclude that numerical constraints usually have the effect of lowering the exponents and that higher exponents are obtained when subjectively appropriate numbers have been assigned either by $E$ or $O$. (The exponent value for the group using fractions in Fig. 3 was the exception.) It is possible that there is a large "stimulus error" in judgments of length, but unless the extraneous contribution of $E$ predetermining $O^{\prime}$ 's response choices is partialed out, any estimate of the "stimulus error" will always be inflated.

\section{References}

Engen, T., \& Levy, N. The influence of standard on psychophysical judgments. Percept. mot. Skills, 1955, 5, 193-197.

Stevens, S. S. On the psychophysical law. Psychol. Rev., 1957. 64, 153-181.

\section{Note}

1. Now at Center for Research in Thinking and Language, Catholic University. 